\title{
Secondary reinforcement measured with unrelated responses in training and testing
}

\author{
JON E. JONSSON, HERBERT FRIEDMAN, and ROBERT A. JOHNSTON \\ College of William and Mary, Williamsburg, Virginia
}

\begin{abstract}
Six groups of rats were trained by feeding paired with different combinations of light and buzzer stimuli. The test was learning to run in a straight runway for the buzzer, light, or food in the goalbox. The group running for buzzer and light stimuli, which reliably preceded feeding, performed as well as the group receiving food. The learning of a new response different from the training response supports an association model of secondary reward rather than a cognitive model. Other groups demonstrated that secondary reinforcement was more a function of the information value of a stimulus than of contiguity.
\end{abstract}

The evidence for secondary reinforcement is extensive but subject to debate. Studies have been criticized for failing to employ appropriate control groups and for not demonstrating either reliable effects or the learning of new responses for secondary rewards. In addition, secondary reinforcement effects can usually be explained in equally plausible ways without invoking an additional construct (see Bolles, 1975; Longstreth, 1975).

Egger and Miller $(1962,1963)$ studied the role of information as a factor in stimuli acquiring secondary reinforcing properties. In training, two distinct stimuli were presented immediately before the primary reinforcement-a tone occurring $.5 \mathrm{sec}$ before a buzzer. In testing, the tone (a reliable indicator of reward) was a more effective reinforcer than the buzzer. However, the dependent measure in testing (barpressing) was the same as the response used in training. Given this confounding, it is not possible to determine whether the informational stimulus would have led to the learning of new responses. A more recent study by Doerries, Silverstein, and Smith (1981) provided additional evidence for the existence of secondary reinforcers with a testing response that was largely, but not totally, unrelated to the training response.

With regard to secondary reinforcement "like" behaviors that occur in the operation of monetary or token reward systems, Bolles (1975) and Longstreth (1971) suggested that since associative theories of secondary reinforcement do not hold up well under critical examination, a cognitive theory (based on the expectation of future rewards) appears to be preferable. However, in animal studies, an expectancy model is

Jon E. Jonsson is currently at the Department of Psychology, University of Southern California, Los Angeles, CA 90089. Requests for reprints should be sent to Herbert Friedman, Department of Psychology, College of William and Mary, Williamsburg, VA 23185. clearly applicable only when the measure of secondary reward involves a response that is the same as or similar to the original training response. The present study, therefore, was designed to test for new learning to a secondary reward in a task totally unrelated to the training task.

\section{METHOD}

Subjects

The subjects were 42 experimentally naive Sprague-Dawley albino rats, 90-120 days of age at the beginning of the study.

\section{Apparatus}

Training of each subject involved one of two special feeding cages. Cage $F$ had a food dish $(9.5 \mathrm{~cm}$ in diameter $\times 5 \mathrm{~cm}$ high) at the rear. A small 3-W white light was located in the left front, $1.2 \mathrm{~cm}$ below the top of the cage. A small electromagnet raised a circular Plexiglas cover off the food dish at random intervals of 1 to $4 \mathrm{~min}$; the cover remained raised $1.5 \mathrm{~min}$ to allow the rat to eat. The second type of cage, Cage NF, had only the $3-\mathrm{W}$ white light and no feeder. The training stimuli employed, depending upon the experimental condition, were 1-sec presentations of the cage light, a $60-\mathrm{dB}$ buzzer, and a tone that was discriminably different from the buzzer.

Tests were carried out in a straight runway measuring $210 \mathrm{x}$ $9.5 \times 12 \mathrm{~cm}$. The startbox and the goalbox each were the same size, $15 \times 15.5 \times 14 \mathrm{~cm}$. The goalbox contained the same type of light and buzzer as the feeding cage. Foot pedals outside the startbox and the goalbox permitted the measurement of running times. Hunter's Choice Dog Ration (Jim Dandy Co., Birmingham, AL) was used for feeding. The average weight of the pellets was $3 \mathrm{~g}$.

\section{Procedure}

Seven groups were employed, each containing six randomly assigned subjects maintained on a $22-\mathrm{h}$ deprivation schedule. All subjects were fed for a 2 -h session in a feeding cage each day for 13 days. Starting on Day 14, the feeding session followed immediately after three massed testing trials in the straight runway. Test sessions were carried out for 12 days. The preliminary training and experimental conditions for each group are described below.

Light and buzzer contingent with feeding-Light and buzzer in runway (LBC-LB). Each subject was put in Cage $F$ and given 
$301.5-$ min feeding periods in a $2-\mathrm{h}$ session. Each raising of the Plexiglass top of the feeder coincided with the termination of a 1 -sec presentation of the light and buzzer. On the following day, each subject was placed in the startbox of the runway. The door of the startbox opened when the subject was facing it. When the rat arrived at the goalbox, the light and buzzer came on for $1 \mathrm{sec}$, and the rat was kept in the goalbox for $10 \mathrm{sec}$.

Light and buzzer noncontingent with feeding-Light and buzzer in runway (LBNC-LB). Each subject was put in Cage NF, which contained only the white light. Food dishes were then placed in the cages, and each subject could eat freely during this 2-h period. Buzzer and white-light presentations were matched to those of the LBC-LB group, but were unrelated to the availability of food. As with the LBC-LB group, each subject had 301.5 -min feeding trials in the training apparatus. On succeeding days, runway testing with the light and buzzer was the same as for the LBC-LB group.

Tone, light, and buzzer contingent with feeding-Light and buzzer in runway (TLBC-LB). This group was trained in the way Group LBC-LB was trained, except that a tone was presented $.5 \mathrm{sec}$ before the light and buzzer onset and was terminated with the light and buzzer on each of the 301.5 -min feeding trials. Each subject had 301.5 -min feeding trials in the training apparatus daily. In the experimental condition, runway training with the light and buzzer was the same as for Group LBC-LB.

Noncontingent feeding-Light and buzzer in runway (NC-LB). This group had no training with the light, buzzer, or tone during the preliminary feeding sessions. The subjects were allowed to eat freely from the food dishes in Cage NF for a 2-h period. In the experimental condition, runway training with the light and buzzer was the same as for Group LBC-LB.

Noncontingent feeding-No light or buzzer in runway (NC-NLB). These animals had no training with the light, buzzer, or tone during the preliminary feeding sessions. The subjects were allowed to eat freely from the food dishes in Cage NF for a 2 -h period. In the experimental condition, no light, buzzer, or tone was presented when the subjects arrived at the goalbox. The subjects were allowed to remain in the goalbox for $10 \mathrm{sec}$.

Noncontingent feeding-Food, light, and buzzer in runway (NC-FLB). These animals had no experience with the light, buzzer, or tone during the preliminary training period. The subjects were allowed to eat freely from the dishes in Cage NF for a 2-h period. In the experimental condition, when the subjects arrived in the goalbox, the light and buzzer were presented for $1 \mathrm{sec}$ and the subjects found five food pellets. The subjects were removed from the goalbox after $10 \mathrm{sec}$.

Noncontingent feeding-Food in runway (NC-F). Preliminary training without light, buzzer, or tone was the same as for Group NC-FLB. The experimental condition was the same as for Group NC-FLB, except that no light or buzzer was presented in the goalbox.

Table 1 summarizes the conditioning treatments and testing conditions for each of the seven groups.

Table 1

Stimuli Presented in Training and Testing

\begin{tabular}{|c|c|c|c|c|c|c|c|}
\hline \multirow[b]{3}{*}{ Group } & \multicolumn{4}{|c|}{ Training } & \multicolumn{3}{|c|}{ Testing } \\
\hline & \multicolumn{2}{|c|}{ Contingent } & \multirow{2}{*}{$\begin{array}{c}\text { Noncon- } \\
\text { tingent } \\
\text { LB }\end{array}$} & \multirow{2}{*}{$\begin{array}{c}\text { No } \\
\text { LB }\end{array}$} & \multirow[b]{2}{*}{ LB } & \multirow{2}{*}{$\begin{array}{l}\text { No } \\
\text { LB }\end{array}$} & \multirow[b]{2}{*}{ Food } \\
\hline & LB & TLB & & & & & \\
\hline LBC-LB & $\mathrm{X}$ & & & & $\mathrm{X}$ & & \\
\hline LBNC-LB & & & $\mathrm{X}$ & & $\mathrm{X}$ & & \\
\hline TLBC-LB & & $\mathbf{X}$ & & & $\mathrm{X}$ & & \\
\hline NC-LB & & & & $X$ & $\mathrm{X}$ & & \\
\hline NC-NLB & & & & $\mathrm{X}$ & & $\mathrm{X}$ & \\
\hline NC-FLB & & & & $\mathrm{X}$ & $\mathrm{X}$ & & $X$ \\
\hline NC-F & & & & $X$ & & $X$ & $X$ \\
\hline
\end{tabular}

Note-See text for explanation of abbreviations.
Table 2

Group Means and Standard Deviations for Each Group for the First 2 and Last 2 Trial Days

\begin{tabular}{llrrrr}
\hline \multirow{2}{*}{$\begin{array}{c}\text { Treatment } \\
\text { Groups }\end{array}$} & & \multicolumn{5}{c}{ Trial Days } \\
\cline { 2 - 6 } LBC-LB & Mean & \multicolumn{1}{c}{14.16} & 48.83 & 18.20 & 16.05 \\
& SD & 28.39 & 50.02 & 6.53 & 5.92 \\
LBNC-LB & Mean & 32.07 & 47.67 & 41.62 & 40.10 \\
& SD & 19.96 & 25.60 & 18.55 & 16.23 \\
TLBC-LB & Mean & 18.82 & 18.07 & 44.37 & 32.92 \\
& SD & 5.34 & 6.59 & 28.98 & 19.19 \\
NC-LB & Mean & 20.36 & 30.26 & 40.74 & 23.34 \\
& SD & 2.54 & 17.07 & 25.93 & 10.49 \\
NC-NLB & Mean & 21.15 & 20.72 & 35.08 & 30.08 \\
& SD & 7.74 & 9.07 & 24.02 & 16.39 \\
NC-FLB & Mean & 24.33 & 25.02 & 15.85 & 15.47 \\
& SD & 15.29 & 11.29 & 6.06 & 3.95 \\
NC-F & Mean & 24.78 & 12.98 & 9.28 & 7.78 \\
& SD & 19.56 & 8.76 & 5.27 & 2.91 \\
\hline
\end{tabular}

Note-See text for explanation of abbreviations.

\section{RESULTS}

Table 2 presents the mean times (in seconds) and standard deviations for each group by trial days. Group LBC-LB (secondary reinforcement) initially ran slowly but increased speed over trials. Several of the other groups (e.g., Group TLBC-LB) began by running quickly but slowed down over trials. The two groups fed in the runway (Groups NC-FLB and NC-F) ran considerably faster than the other groups.

Figure 1 compares Group LBC-LB with its three most cogent control groups.

A split-plot factorial design (Kirk, 1968) was used to analyze the data (treatment groups $x$ trial days), with the partial eta coefficient (Cohen, 1973) employed as a measure of size of effect. Due to the death of one Group NC-LB subject, an unweighted means solution was used. The effect for treatment groups $[\mathrm{F}(6,34)=$ $5.03, \mathrm{p}<.001$, eta $=.61]$ and the groups $\mathrm{x}$ trials interaction $[F(66,374)=1.89, \mathrm{p}<.001$, eta $=.34]$ were both significant. The effect for trials $[\mathrm{F}(11,374)=1.40$, $\mathrm{p}<.17$, eta $=.11]$ was not significant.

A separate analysis tested group differences on the first 2 and on the last 2 trial days. In the first analysis, Group LBC-LB was compared with Groups LBNC-LB and TLBC-LB. Using Dunn's conservative $t$ values (Kirk, 1968), no significant difference was found between these groups over the first 2 trial days $[\mathrm{t}(16)=.918$, $\mathrm{p}>.10, \mathrm{rm}<.25$ (Friedman, 1982)]. However, a significant difference was found between these groups over the final 2 trial days $[\mathrm{t}(16)=-3.61, \mathrm{p}<.01, \mathrm{rm}>.65]$. The second comparison made was between Group LBCLB and Group NC-FLB. No significant difference was found over the first 2 trial days $[t(10)=1.17, p>.10$, $\mathrm{rm}<.10]$ or during the final 2 trial days $[\mathrm{t}(10)=.49$, $\mathrm{p}>.10]$, in both cases using Dunn's conservative values. In a comparison between Group LBC-LB and Groups 


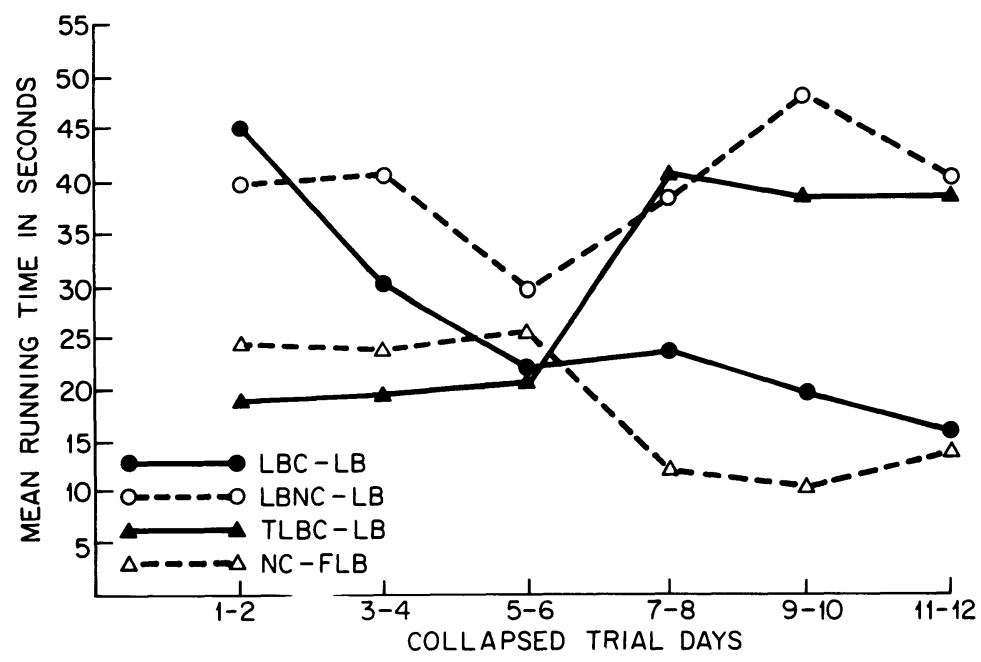

Figure 1. Mean running time across trial days for the main experimental groups.

NC-LB and NC-NLB, no difference was found over the first 2 trial days $[t(16)=1.30, p>.10]$, but a significant difference was found over the last 2 trial days. $[\mathrm{t}(16)=-2.69, \mathrm{p}<.05, \mathrm{rm}>.55]$, again using Dunn's values.

\section{DISCUSSION}

Direct evidence for a secondary reinforcement effect in the learning of a new response unrelated to the training response was provided by Group LBC-LB, which ran more quickly than any of the other groups except those receiving food in the goalbox. Group NC-FLB (food in the runway) performed essentially the same as Group LBC-LB over the final 2 trial days, showing that the secondary reinforcement approached the potency of the primary reinforcement. The increase in running speed across trial days for Group LBC-LB when no food was ever provided in the goalbox is consistent with an associative model of secondary reward. These results do not fit cognitive models, which would require that the testing response be similar to the training response.

Group LBC-LB did not differ significantly from Group TLBCLB at the start of testing, but on the final trial days Group LBCLB ran significantly faster. Group TLBC-LB received the same treatment as Group LBC-LB, with the addition of a tone immediately preceding the light and buzzer. This tone prevented the redundant light and buzzer from acting as a secondary reinforcer; presumably, the tone itself would have been effective if it had been presented in the runway. This finding supports Egger and Miller's $(1962,1963)$ interpretation of conditioned reinforcement, which emphasizes the information value of a stimulus, rather than contiguity.

Two minor aspects of Table 2 deserve comment. First, the light and buzzer in the runway slowed the running of the groups that had not been exposed to these stimuli in training; this slowing was due possibly to distraction. For example, Group NCFLB ran considerably slower than Group NC-F: these groups differed only with respect to the stimuli experienced in the runway. Second, although Group LB-LBC ran slower than Groups NC-LB and NC-NLB at the beginning of training, this difference was not significant. However, Group LBC-LB ran significantly faster than those two groups on the final 2 trial days.
For all but Group NC-FLB and NC-F, the three daily massed runway trials did involve extinction in terms of the primary reinforcement (food) and therefore may have produced frustration during testing. This frustration should have been minimal, however, since immediately after the experimental trials, each subject was taken to a food cage. This procedure is analogous to that employed by Saltzman (1949) and allows the measurement of secondary reinforcement without the confounding effects of frustration (see Lott, 1967).

\section{REFERENCES}

Bolles, R. C. (1975). Motivation (2nd ed.). New York: Harper \& Row.

Cohen, J. (1973). Eta-squared and partial eta-squared in fixed factor ANOVA designs. Educational and Psychological Measurement, 33, 107-112.

Doerries, L. E., Silverstein, A., \& Smith, N. F. (1981). Acquisition and retention of conditioned reinforcement as a function of variability in training setting and distribution of testing. Hartford, CT: Eastern Psychological Association.

EgGer, N. D., \& Miller, N. E. (1962). Secondary reinforcement in rats as a function of information value and reliability of the stimulus. Journal of Experimental Psychology, 64, 97-104.

Egger, N. D., \& Miller, N. E. (1963). When is a reward reinforcing? An experimental study of the information hypothesis. Journal of Comparative and Physiological Psychology, 56, 132-137.

Friedman, H. (1982). Simplified determinations of statistical power, magnitude of effect and research sample sizes. Educational and Psychological Measurement, 42, 521-526.

KIRK, R. E. (1968). Experimental design: Procedures for the behavioral sciences. Monterey, CA: Brooks/Cole.

LONGSTRETH, L. E. (1971). A cognitive interpretation of secondary reinforcement. Nebraska Symposium on Motivation, 19, 33-80.

LOTT, D. F. (1967). Secondary reinforcement and frustration: A conceptual paradox. Psychological Bulletin, 67, 197-198.

Saltzman, I. J. (1949). Maze learning in the absence of primary reinforcement: A study of secondary reinforcement. Journal of Comparative and Physiological Psychology, 42, 161-173.

(Manuscript received for publication March 19, 1984.) 